\author{
Revue \\ de 'histoire \\ Revue de l'histoire des religions \\ des religions \\ $2 \mid 2008$ \\ La mort et l'émotion. Attitudes antiques
}

\title{
Le deuil à Rome : mise en scène d'une émotion
}

The staging of an emotion: the example of mourning in Rome

\section{Francesca Prescendi}

\section{CpenEdition}

Journals

Édition électronique

URL : http://journals.openedition.org/rhr/6123

DOI : $10.4000 /$ rhr.6123

ISSN : 2105-2573

Éditeur

Armand Colin

Édition imprimée

Date de publication : 1 avril 2008

Pagination : 297-313

ISSN : 0035-1423

\section{Référence électronique}

Francesca Prescendi, «Le deuil à Rome : mise en scène d'une émotion », Revue de l'histoire des religions [En ligne], 2 | 2008, mis en ligne le 01 avril 2011, consulté le 19 avril 2019. URL : http:// journals.openedition.org/rhr/6123; DOI : 10.4000/rhr.6123 
FRANCESCA PRESCENDI

Université de Lausanne et de Genève

\section{Le deuil à Rome : mise en scène d'une émotion}

Le deuil dans la littérature romaine est considéré comme une forme particulière de tristesse. Il s'agit d'une émotion, qui pourtant est rarement décrite dans son aspect privé et intime. Quand on parle de deuil, c'est plutôt l'aspect rituel qui est mis en avant. Cette émotion en effet est ritualisée et mise en scène publiquement lors des funérailles. La fonction de ce rite est de canaliser la douleur pour la contrôler afin qu'elle ne sombre pas dans le paroxysme. Si la ritualisation du deuil a un effet cathartique, elle est en même temps aussi le moteur d'autres émotions qui surgissent au moment des funérailles. Le rite est un élément qui permet de passer d'une dimension affective et intime à un fait social et public, il est un point de départ et d'arrivée des émotions.

\section{The staging of an emotion: the example of mourning in Rome}

Mourning in the Ancient Roman literature is considered a peculiar sort of sadness, but it is rarely depicted as a private emotion. Speaking of mourning mostly implies a reference to its ritual side. This emotion is ritualised and staged during the funerals. The function of this rite is to canalize the sorrow as to keep it in control, avoiding paroxysm. The ritualism has hence a cathartic sense, but at the same time it gives way to other emotions rising during the funerals. The ritual allows proceeding from a domestic and intimate dimension to a social and public event: it is a departure and a final goal for the emotions. 


\section{1/ LE DEUIL : ÉMOTION ET RITE}

Dans son livre récent sur les émotions dans le monde grec, David Konstan ${ }^{1}$ explique que le deuil, à la différence d'autres émotions telles que la colère, la pitié ou l'indignation, n'est pas lié à un sentiment d'injustice ou de blessure. Selon Konstan, la perte d'une personne aimée ne nécessite guère d'évaluation. Point n'est besoin du passage par cette étape bien connue par les spécialistes des émotions : l'appraisal. Pour cela, le deuil se distingue nettement d'autres émotions comme la colère, par exemple, provoquée par une offense, qui nécessite, de la part de l'offensé, une réelle évaluation du « dommage ». Dans cette évaluation, les intentions de l'offensant, son statut social et d'autres éléments circonstanciels jouent un rôle. L'intensité de l'émotion ressentie par l'individu «blessé » dépend de cette évaluation. Dans le cas de la peur, toujours selon Konstan, l'individu doit évaluer le danger effectif que l'adversaire représente. La particularité du deuil est que l'individu frappé par la mort d'un conjoint ressent une émotion indépendante des intentions d'un possible adversaire, de sa puissance ou de son statut social. Ces différentes raisons, en plus du fait que le deuil (lupê, penthos) n'est pas mentionné dans la liste des pathé du deuxième livre de la Rhétorique d'Aristote, font que Konstan propose de ne pas inclure le deuil dans la liste des affects que les Grecs auraient reconnus comme émotions.

Pour le monde romain, le discours se présente d'une manière différente. Si pour Pline le deuil (luctus) est une des qualités qui fait la différence entre l'homme et les animaux ${ }^{2}$, pour Cicéron, il trouve sa place parmi d'autres affects qu'il définit comme perturbationes et qu'il rattache à une émotion principale $:$ le « chagrin » $(\text { aegritudo })^{3}$.

1. Konstan, 2006.

2. Plin., nat., 7, 4-5 : uni animantium luctus est datus (« à l'homme seulement parmi tous les êtres animés a été donné le deuil »), traduction de Robert Schilling, Les Belles Lettres, Collection des Universités de France (CUF), Paris, 1977.

3. Cic., Tusc., 4, 16 : sed singulis perturbationibus partes eiusdem generis plures subiciuntur, ut aegritudini invidentia utendum est enim docendi causa verbo minus usitato, quoniam invidia non in eo qui invidet solum dicitur, sed 
Il semble donc que le deuil est reconnu par les Romains comme une émotion, c'est-à-dire un affect capable de bouleverser, de créer une perturbatio dans l'esprit humain. Pour préciser de quel type d'aegritudo il s'agit, Cicéron ajoute une information importante : il est rattaché à la "tristesse pour la mort "prématurée" (acerbus) de quelqu'un qui fut cher ${ }^{4}$. Le mot acerbus, utilisé souvent pour désigner la mort des enfants (équivalent au grec aoros), revêt ici probablement plus le sens général de décès touchant tous ceux qui n'ont pas atteint un âge vénérable, un âge «adapté » à la mort. J. Humbert, traducteur du livre des Tusculanae Disputationes de Cicéron édité aux Belles Lettres en 1968, comprend, à juste titre, acerbus dans l'acception de « cruel » ${ }^{5}$. Sénèque (cons. Marc. 19,1), en essayant de définir en quoi consiste la douleur du deuil, parle de desiderium ( « regret») pour la personne disparue.

Ces différents passages permettent une description nuancée de ce que ressent une personne endeuillée : il s'agit du profond désespoir suscité par une mort considérée comme prématurée, imprévisible et injustifiée, et ce sentiment est lié à de la nostalgie. Ces considérations permettent de repenser l'importance de l'appraisal qui suit la constatation de la mort d'un conjoint. S'il n'y a pas une évaluation des intentions d'un possible adversaire, ni du danger qu'il pourrait représenter, comme dans le cas de la colère ou de la peur, cela ne signifie pas qu'il n'y ait pas de réaction. Dans le cas du deuil, l'appraisal consiste à se mesurer à l'idée de précarité et d'arbitraire de la vie humaine. Tout comme la personne victime d'une injure, l'individu endeuillé se sent victime mais, à la différence de l'offensé, la blessure n'est pas provoquée par un autre être humain : elle est conséquence de la condition humaine. On se laisse emporter par la colère, on sombre dans une profonde douleur.

etiam in eo cui invidetur, aemulatio, obtrectatio, misericordia, angor, luctus, maeror, aerumna, dolor, lamentatio, sollicitudo, molestia, adflictatio, desperatio, et si quae sunt de genere eodem.

4. Cic., Tusc., 4, 18 : luctus aegritudo ex eius qui carus fuerit interitu acerbo (texte établi par Georges Fohlen et trad. par Jules Humbert, t. 2, III-V, Les Belles Lettres, CUF, Paris, 1968).

5. La même interprétation est proposée par Graver (2002). 
Une description utile pour l'étude de l'appraisal lié à la prise de conscience de la mort se trouve au chant 2, 20-28 de la Pharsale de Lucain. Pour illustrer la situation difficile de la ville de Rome après le début de la guerre civile entre César et Pompée, le poète fait une comparaison avec une famille en deuil.

Alors on retint ses plaintes, tous furent pénétrés d'une douleur profonde et silencieuse. Ainsi, au début d'un deuil, les familles étourdies se taisent, quand les corps, qui n'ont pas encore été appelés (conclamare), sont couchés et la mère, qui n'a pas encore incité les bras de ses servantes au planctus avec les cheveux dénoués, serre les membres raidis par la fuite de la vie, les traits du visage inanimés et les yeux menaçant dans la mort. Ce n'est pas encore la douleur, ce n'est plus la crainte : elle s'incline affolée et s'étonne de son malheur' ${ }^{6}$.

La première phrase de ce passage fait encore partie de la description de la ville pendant la guerre civile. La ville est décrite comme envahie d'un dolor sine voce. C'est cette douleur qui constitue l'élément de comparaison avec la famille en deuil. La phase du deuil est celle qui suit immédiatement le décès. C'est l'expression funere primo, ne signifiant pas les premières funérailles mais la première phase du funus, qui l'indique. La famille est désignée par le substantif pluriel domus auquel s'ajoute un adjectif très expressif : attonitae. Cet adjectif signifie, dans son sens premier, «frappé par la foudre » et, dans son sens dérivé, «étourdi, abasourdi ». Lucain décrit alors la surprise de la famille causée par le choc émotionnel du décès. Ce moment de silence précède, dit le texte, la conclamatio, c'est-à-dire l'appel répété du nom du défunt, pratique qui sert selon les anciens à constater que le décès est effectif ${ }^{7}$. Ensuite, l'attention se concentre sur la mère de famille : elle est décrite dans le moment qui précède celui où elle dénouera ses cheveux (nec mater crine soluto) et appellera ses servantes pour faire le planctus (exigit ad

6. Lucan., bell. civ., 2, 20-28: Tum questus tenuere suos, magnusque per omnes/ Erravit sine voce dolor. Sic funere primo/Attonitae tacuere domus, cum corpora nondum/ Conclamata iacent nec mater crine soluto/ Exigit ad saevos famularum bracchia planctus,/ Sed cum membra premit fugiente rigentia vita/ Vultusque exanimes oculosque in morte minaces;/ Necdum est ille dolor nec iam metus: incubat amens/ Miraturque malum (trad. A. Bourgery, 1997, modifiée, $2^{\mathrm{e}}$ édition revue et corrigée par Paul Jal, Les Belles Lettres, CUF, Paris, 1997).

7. Serv., Aen., 6, 218. 
saevos famularum bracchia planctus). Elle embrasse le corps du mort pour prendre conscience du décès : c'est le moment de la constatation. Au vers 27, la phrase necdum est ille dolor nec iam metus est très significative. La phase du deuil décrite ici est celle qui suit un état de peur provoqué par la pensée d'une mort possible. En même temps, cette phase précède la douleur, un sentiment qui pourra venir seulement après la prise de conscience du décès. L'état de stupeur silencieuse se situe donc entre ces deux moments. La mère y est amens : « elle a perdu ses esprits et s'étonne du mal qu'elle a devant les yeux (miraturque malum) $»^{8}$.

Lucain, dans son analyse de la réaction psychologique de la mère endeuillée, fait exception. La plupart des auteurs latins, pour décrire des femmes affligées, dépeignent les gestes codifiés de la lamentation. À l'annonce d'une mort, celles-ci se déchirent les joues, s'arrachent les cheveux, les salissent avec de la cendre, se frappent la poitrine dénudée ${ }^{9}$ jusqu'à en faire sortir le sang ou le lait ${ }^{10}$. Un parallèle figuré de ces descriptions littéraires se trouve dans une scène du relief des Haterii (figure 1) où des femmes, derrière le lit funèbre sur lequel une défunte est couchée (scène de prothésis), se sont dénudé la poitrine pour se frapper. En bas, sous le lit, d'autres personnes sont représentées qui se touchent la poitrine en faisant un geste de lamentation. Par ailleurs, Servius (Aen. 11, 211) explique que l'attitude de se frapper pour le deuil s'appelle planctus. Ce planctus, de même que la lamentation, peut se comprendre comme

8. La phase émotionnelle vécue par cette femme peut être comparée à la propatheia des Stoïciens, c'est-à-dire au moment dans lequel le stimulus émotionnel est perçu mais où il n'a pas encore provoqué une réaction, Graver (2008) (je remercie l'auteur de m'avoir permis de lire ces considérations avant la parution du livre). Sur cette réaction au stimulus, il y a l'intéressante étude de Philippe Borgeaud (2007) qui compare la phase de l'évaluation d'une émotion aux procédures politiques par lesquelles le Sénat romain décide d'accepter ou non un prodige.

9. Catul. 64, 350 ; Prop. 2, 13, 27 ; Tib. 1, 1, 67-68 ; Verg., Aen., 12, 602-608, Ov., trist., 3, 3, 51. Sur les mêmes attitudes en Égypte, cf. la contribution de Y. Volokhine dans ce même volume.

10. Serv., Aen., 3, 67; 5, 78 affirme que le lait et le sang servent comme aliment pour les esprits des défunts. Cet aspect a été très bien analysé par Corbeill 2004, 86 ss. 


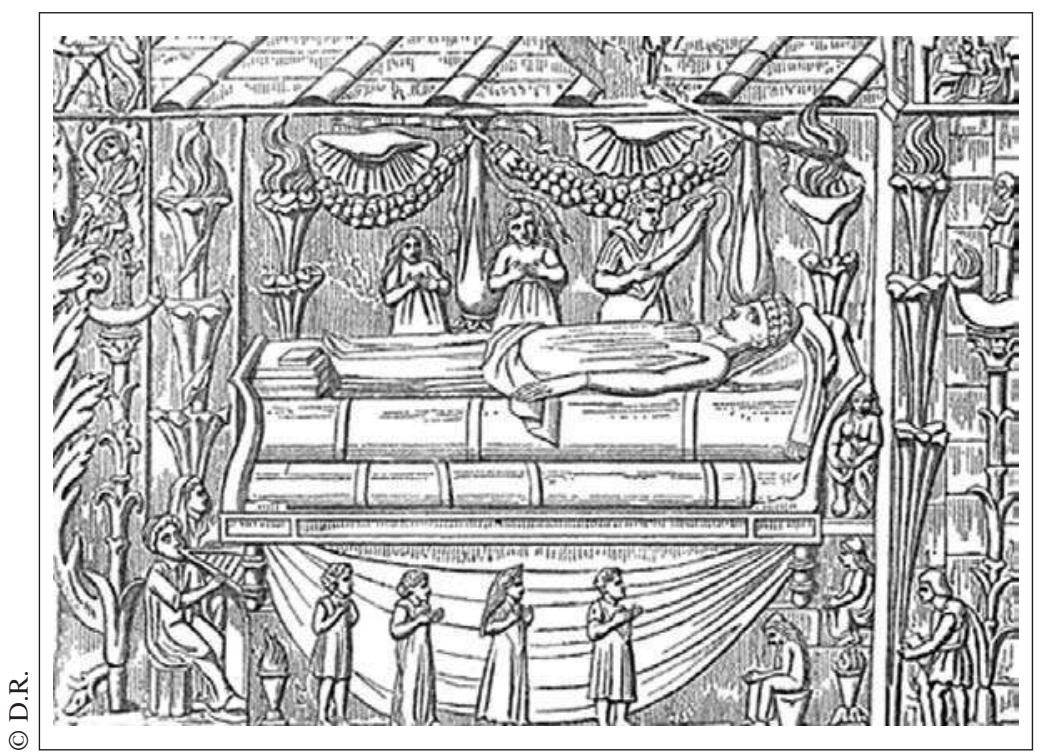

Fig. 1. Relief des Haterii, (vers 110 ap. J.-C.), Musei Vaticani, Roma. (Dessin tiré de Ch. Daremberg, Edm. Saglio, Dictionnaire des Antiquités grecques et romaines, tome 2, $1896 \mathrm{sv}$ funus, p. 1389, fig. 3360).

expression privée et spontanée mais aussi comme une manifestation organisée, collective et rituelle. Nous retrouvons cet aspect communautaire dans la mise en place d'une manifestation chorale, admirablement bien décrite, dans les Troyennes de Sénèque ${ }^{11}$. Frappée par la mort d'Hector, Hécube incite un groupe de femmes (le chœur) à accomplir les gestes de la lamentation avec elle :

LE CHEUR. - Allez faire retentir vos douleurs ; lève ta main marquée par le malheur ; nous, (80) masse insignifiante, nous suivrons notre maîtresse : nous sommes bien dressées aux lamentations.

HÉCUBE. - Fidèles compagnes de notre malheur, dénouez votre chevelure, que sur vos cous accablés flottent vos cheveux souillés par la cendre encore chaude de Troie. Que votre troupe se tienne prête, bras en l'air ; faites tomber vos vêtements, puis attachez-les en laissant

11. Sen., Tro., 79-105 (traduction de François-Régis Chaumartin, Les Belles Lettres, CUF, Paris, 1996). 
vos corps découverts jusqu'au ventre. Pour quel mariage (90) couvres-tu les seins d'un voile, pudeur, quand tu es captive ? Que votre écharpe serve de ceinture à vos tuniques défaites, que vos mains furibondes soient libres pour répandre sur vos corps une pluie de coups. Vous me plaisez en cet état, vous me plaisez: je reconnais ma troupe de Troyennes. Que reviennent, aujourd'hui encore, les (95) anciennes lamentations ; mais allez au-delà de votre manière ordinaire de pleurer : c'est Hector que nous pleurons.

LE CHEUR. - Toutes nous avons dénoué notre chevelure (100) mise à mal par tant de deuils, nos cheveux pendent, libres de leur nœud et sur nos visages est répandue la cendre chaude ; remplissez-en vos mains ; voilà ce que nous avons le droit d'emporter de Troie. Nos vêtements tombent de nos épaules découvertes et, attachés par un nœud, (105) couvrent le bas de nos flancs ; maintenant nos poitrines appellent nos mains.

On voit dans ce passage une femme accablée par la douleur qui appelle d'autres femmes pour partager son émotion. Celle-ci prend alors la forme d'un rite collectif. L'aspect rituel du deuil est souligné par le fait qu'en plus des femmes et des servantes de la famille, les lamentations sont confiées (surtout pour les familles riches) à des praeficae, c'est-à-dire des professionnelles. Ces femmes étaient choisies pour leur voix remarquable ${ }^{12}$ et étaient payées pour se rendre ${ }^{13}$,

12. Varro, vit. popul. Rom. (ap. Nonius 212 L. ; fr. 110 Benedetto Riposati, M. Terenti Varronis, De vita populi Romani, Milano, 1939) : NENIA, ineptum et inconditum carmen, quod a conducta muliere, quae praefica diceretur, $\hat{s}$, quibus propinqui non essent, mortuis exhiberetur. Varro de Vita Populi Romani lib. IV : ibi a muliere, quae optuma voce esset, perquam laudari; dein neniam cantari solitam ad tibias et fides... («La nenia est un chant brut et informe, qui était récité par une femme rétribuée appelée praefica pour des défunts qui n'auraient pas de parents proches. Varron au livre 4 de son de Vita Populi Romani : là le mort était loué de manière satisfaisante par une femme douée d'une voix remarquable; ensuite on avait l'habitude de chanter la nenia au son de la tibia - hautbois - et de la lyre..., trad. personnelle). À propos de la musique Lucien., luct., 19 affirme que les gémissements et les coups sur la poitrine étaient rythmés par le son de la flûte.

13. Lucil., $22(27,18)$ ap. Non. 93 L. (De compendiosa doctrina libros $X X$, éd. Wallace M. Lindsay, K.G. Saur, München, 2003) : mercede quae conductae flent alieno in funere, praeficae multo et capillos scindunt et clamant magis ( « Payées pour le faire, les praeficae pleurent lors des funérailles d'autrui, s'arrachent les cheveux et crient abondamment », trad. personnelle). Dans le bois de Libitina se trouvait des entreprises de pompes funèbres, Scheid (2004). 
depuis le bois de Libitina, au seuil de la maison du mort ${ }^{14}$. Elles se mettaient alors à la tête (praeficere) du groupe des servantes afin de leur montrer comment se lamenter ${ }^{15}$. Les rares informations que l'on rencontre semblent indiquer que ces femmes étaient payées pour réciter des chants funèbres ${ }^{16}$. Ces chants, les neniae, devaient être des lamentations topiques, non personnalisées, qui étaient

14. Brigger et Giovannini (2004, p. 197), sur la base des textes littéraires et de l'iconographie arrivent à la conclusion qu'il y a deux moments distincts pour la lamentation grecque : pendant la prothésis, les membres de la famille se lamentent de manière spontanée devant le cadavre auquel des personnes étrangères rendent visite ; pendant le cortège funèbre, les personnes extérieures à la famille font la lamentation rituelle. À Rome, les descriptions ne sont pas assez abondantes pour pouvoir établir avec précision qui sont les personnes qui agissent pendant les différents moments des funérailles. Les bas-reliefs (fig. 1 et 3) montrent des personnes qui font la lamentation, mais il est difficile de dire s'il s'agit de femmes de la famille ou de praeficae. D'après Varron (ling., 7, 70), on sait que les praeficae interviennent devant la maison du mort (quae ante domum mortu, pour le passage en entier, $c f$. note suivante). On pourrait imaginer qu'elles chantent pendant la prothesis. Le travail de Brigger et de Giovannini montre que la prothésis dans le monde grec et étrusque a lieu dans une tente devant la maison du mort. On ne sait pas si le même usage existait aussi à Rome. Dans ce cas, l'indication de Varron « devant la maison » pourrait faire allusion à ce moment.

15. Varro, ling. 7, 70: <praefica > dicta, ut Aurelius scribit mulier ab luco quae conduceretur, quae ante domum mortui laudis eius caneret [...] Claudius scribit: "quae praeficeretur ancillis, quemadmodum lamentarentur, praefica est dicta ». ( On appelle praefica, comme l'écrit Aurelius, la femme qu'on prenait à solde du bois [de Libitina], qui devant la maison du mort chantait les louanges de celui-ci. [...] Claudius écrit : "la praefica est appelée ainsi parce qu'elle est à la tête du groupe des servantes auxquelles elle apprend comment se lamenter". » traduction personnelle; texte: M. Terenti Varronis «De lingua latina » quae supersunt; accedunt gramaticorum Varronis librorum fragmenta, recensuerunt Georgius Goetz et Fridericus Schoell, Teubner, Leiprig, 1910).

16. Kudlien (1995) ; Sterbenc Erker, 2004, p. 269 ss. Le passage de Varro, vit. popul. Rom. (ap. Nonius 212 L. ; fr. 110 Riposati), dont j'ai cité une partie auparavant fait allusion au fait que les neniae ont existé jusqu'à l'une des trois guerres puniques: haec mulier vocitata olim praefica usque ad Poenicum Bellum. Le passage est difficile à interpréter. Signifie-t-il qu'après une guerre punique des femmes payées pour pleurer avaient été interdites ? Ou que ces femmes ne s'appelaient simplement plus praeficae ( $c f$. la discussion chez Kudlien, 1995, p. 181) ? Ou encore qu'elles ne chantaient plus les neniae (Riposati, 1939, p. 225-226)? 
rythmées au son d'instruments musicaux ${ }^{17}$. La praefica disposait peut-être de quelques modèles ou trames qu'elle adaptait selon les circonstances, sans rien ajouter d'essentiel ${ }^{18}$. Dans son traité sur le deuil, Lucien de Samosate (luct. 20) qualifie d' «acteur » (sunagonistes) le poète appelé à composer le thrène, c'est-à-dire le chant funèbre qui correspond à la nenia. De même, les praeficae peuvent être qualifiées d'actrices par la mise en scène qu'est le rite du deuil ${ }^{19}$.

Les éléments observés jusqu'à présent nous ont permis de clarifier l'imbrication très étroite de deux aspects du deuil, celui de l'émotion intime et celui du rite collectif du planctus. Il faut maintenant se demander pourquoi cette émotion devient rite.

\section{2/ Pourquoi Ritualiser une Émotion?}

Le livre de E. De Martino, Morte e pianto rituale ${ }^{20}$, bien que publié voici déjà une trentaine d'années, reste indispensable pour comprendre le deuil dans les sociétés anciennes et traditionnelles de la Méditerranée. À partir de recherches menées en Italie du sud, De Martino observe que l'expression du deuil se manifeste en plusieurs étapes. D'abord, le sentiment qui suit immédiatement le décès est une stupeur qu'il appelle ebetudine stuporosa : c'est la prise de conscience « hébétée » du décès. Puis, vient la manifestation spontanée de la douleur (planctus irrelativo). Et enfin, commence la lamentation rituelle (planctus rituale) mise en scène par un groupe de femmes ayant pour fonction d'empêcher la personne

17. Les Romains connaissent aussi une déesse Nenia qui avait probablement un lieu de culte près de la porte du Viminal (Fest. 154 L. ; Paul-Fest. 155 et 157 L.). Perfigli, 2004, p. 33, explique que la déesse Nenia comme d'autres divinités fonctionnelles représentent un aspect culturel significatif.

18. C'est peut-être pour cela que la nenia est définie par Nonius $212 \mathrm{~L}$. (De compendiosa doctrina libros XX, éd. Wallace M. Lindsay, K.G. Saur, München, 2003) comme ineptum et inconditum carmen, c'est-à-dire comme chant « brut et informe ».

19. Kudlien, 1995, p. 185-186.

20. De Martino (1975). 
affligée de sombrer dans un état de douleur irréversible. La femme touchée par la mort d'un conjoint, qui se laisse dans un premier temps aller à exprimer son émotion de façon incontrôlée, est invitée à se lamenter de façon plus régulière et à partager ses pleurs avec d'autres personnes du même sexe. En somme, la ritualisation de la lamentation s'affirme comme un moyen de canaliser l'émotion et de l'empêcher de se transformer en état mental durable. Dans son analyse, De Martino se réfère à plusieurs textes classiques. Il omet celui de Lucain mais en fournit, sans le vouloir, une analyse pertinente. Lucain parle, comme nous l'avons vu, de la stupeur silencieuse qui précède l'organisation de la lamentation rituelle et collective. Cette lamentation ne commence qu'à partir du moment où la femme demande aux servantes de pleurer avec elle. Le planctus rituel auquel Lucain fait allusion, et qui apparaît plus clairement dans le passage de Sénèque, se montre, certes, comme un moyen de contrôler l'émotion, mais aussi de la partager et de la répartir de façon à ce que la violence soit réduite, comme le souligne De Martino.

Après avoir observé les rites, tournons notre regard vers les mythes qui réfléchissent sur les limites extrêmes du deuil. L'histoire de Niobé est emblématique. En effet, cette femme eut le courage et l'audace de défier les dieux. Elle osa déclarer que sa progéniture (sept filles et sept garçons) était beaucoup plus importante que celle de Latone et, par la suite, elle voulut dissuader les femmes d'honorer la déesse. Ce faisant, elle suscita la colère de Latone et de ses enfants, Apollon et Artémis, qui se vengèrent en massacrant la descendance directe de Niobé. Celle-ci, ayant méprisé la puissance divine, en est devenue victime. Elle doit supporter la douleur d'assister à la disparition de sa famille. Ovide (met. 6, 301-312) la décrit ainsi :

« Ayant perdu toute sa famille, ses fils, ses filles et son époux, elle tombe assise entre leurs corps inanimés, figée par la souffrance (deriguit malis) ; le vent n'agite plus ses cheveux, le sang ne colore plus son visage; ses yeux s'immobilisent au milieu de sa face désolée ; il n'y a plus rien de vivant dans ses traits. Sa langue même se glace à l'intérieur de son palais durci et tout mouvement s'arrête dans ses veines ; son cou ne peut plus fléchir, ses bras ne peuvent faire un geste, ni ses pieds s'avancer ; jusque dans ses entrailles elle n'est plus que pierre. Elle pleure pourtant ; un vent impétueux, l'enveloppant d'un tourbillon, l'a emportée 
dans sa patrie ; là, fixée sur le sommet d'une montagne, elle se fond en eau et aujourd'hui encore ce bloc de marbre verse des larmes. ${ }^{21}$ »

La douleur fait entrer Niobé dans un état psychologique irréversible semblable à l'immobilité de la mort qui est ici explicitement évoquée : nihil est in imagine vivum. Cette immobilité de la douleur est représentée dans le langage mythique par la métamorphose en pierre. L'iconographie grecque en donne une image émouvante. Sur une hydrie campanienne (fig. 2), Niobé est représentée au moment où son corps se transforme. La pierre a atteint la taille et le bas du corps est devenu statue, tandis que les bras expriment encore des gestes de douleur $^{22}$. Niobé, qui a défié les dieux, reste prisonnière de sa douleur qui se fige dans l'éternité et l'immuabilité d'une pierre. Le mutisme de la pierre est symptomatique de son état de deuil irréversible.

Le mythe de la déesse Déméter/Cérès au moment du rapt de Perséphone offre un autre exemple. Après avoir pleuré la disparition de sa fille, elle s'enferme dans un long silence. Ovide (fast. 4, 503-506) la décrit après son débarquement à Eleusis :

« Là, pour la première fois, elle s'assit, plongée dans sa tristesse, sur un rocher glacé : les descendants de Cécrops l'appellent encore aujourd'hui le rocher de l'affliction (tristis). Pendant plusieurs jours elle resta immobile à l'air libre, sans broncher ni sous la lune ni sous la pluie » (trad. Robert Schilling, 1992).

Ensuite, alors qu'un homme âgé qui passe par là l'invite à se rendre chez lui et qu'une petite fille qui l'accompagne appelle la déesse «mère », « une goutte brillante (gutta lucida), semblable à une larme (car les dieux ne peuvent pas verser de larmes), tombe

21. Ov., met., 6, 301-312: orba resedit/ exanimes inter natos natasque virumquel deriguitque malis; nullos movet aura capillos, / in vultu color est sine sanguine, lumina maestis/ stant inmota genis, nihil est in imagine vivum./ ipsa quoque interius cum duro lingua palato/ congelat, et venae desistunt posse moveri; / nec flecti cervix nec bracchia reddere motus / nec pes ire potest; intra quoque viscera saxum est./ flet tamen et validi circumdata turbine venti/ in patriam rapta est: ibi fixa cacumine montis/ liquitur, et lacrimas etiam nunc marmora manant (texte établi et traduit par Georges Lafaye, T. 2, VI-X, $2^{\mathrm{e}}$ tirage de la $7^{\mathrm{e}}$ éd. revue et corr. par Henri Le Bonniec, Les Belles Lettres, CUF, Paris, 2000).

22. À voir aussi d'autres images dans le Lexicon Iconographicum Mythologiae Classicae (LIMC), 6, 1 . 


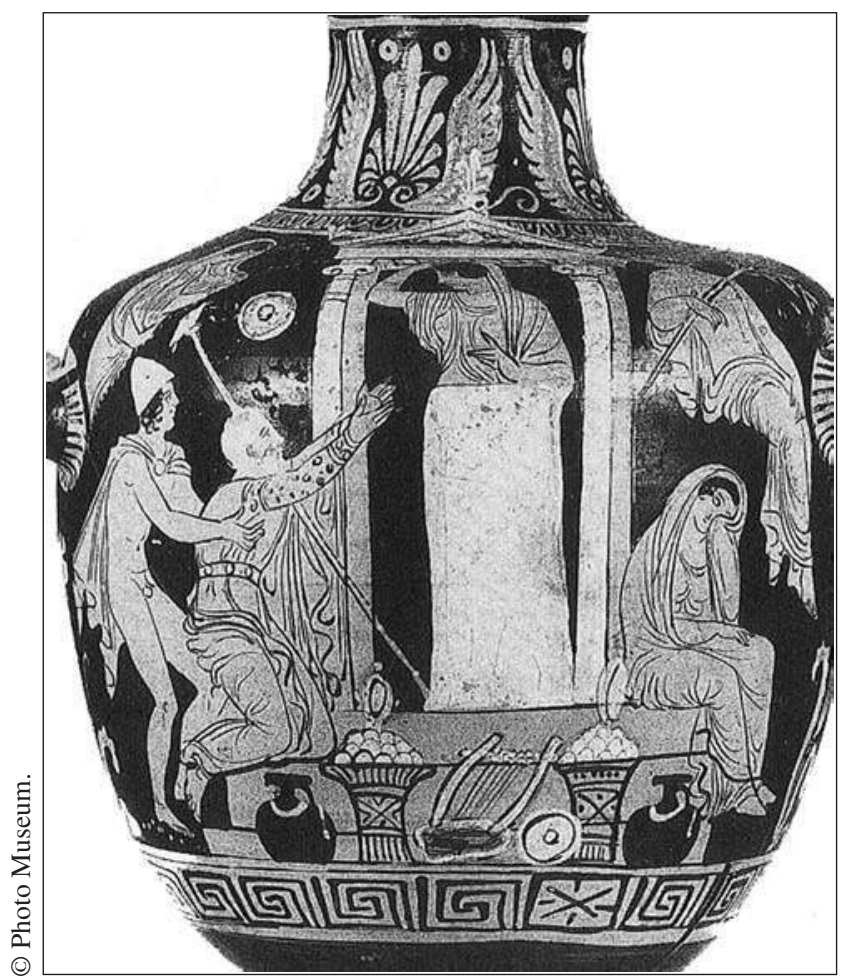

Fig. 2. Hydria, campan., Sydney, Nicholson Museum of Antiquities 71.01 (LIMC vol. 6, planche 610, fig. 11). Vers 340-330 av. J.-C.

sur son sein tiède » (fast. 4, 521-522). La profonde tristesse de Déméter/Cérès est ainsi décrite, dans ce passage aussi, comme une attitude d'immobilité. La pierre de la tristesse rappelle, par sa symbolique, la pierre dans laquelle se transforme Niobé. Déméter/ Cérès quitte l'état d'apathie et reprend vie grâce à la rencontre de deux personnes qui l'amènent chez elles, dans une maison, c'est-àdire au sein d'une vie quotidienne et communautaire.

Les deux récits mythiques de Niobé et de Déméter/Cérès montrent comment le deuil peut entraîner un individu vers un état psychologique potentiellement dangereux, si l'émotion n'est pas limitée et prise en charge par la collectivité. Les analyses que De Martino a faites en se basant sur des documents anciens et traditionnels semblent 
donc constituer une grille de lecture du rite du planctus et de la lamentation qu'il suscite dans la société romaine.

Après avoir examiné ces mythes, retournons au rite spectaculaire du deuil qui peut être comparé à un autre rite de mise en scène d'une émotion: la supplicatio ${ }^{23}$. À la différence de la plupart des rites romains - ne laissant pas percevoir des émotions - la supplicatio peut être définie avec B. Linke ${ }^{24}$ comme une "hochemotionalisierte Form » afin de se procurer la bienveillance divine. Dans le passage de Lucain, la famille en deuil est représentative d'une ville accablée par la guerre civile, où les femmes feront des supplicationes. Lucain perçoit la ressemblance entre lugentes et supplicantes. Les suppliantes sont en effet décrites ainsi ${ }^{25}$ :

« La matrone a déposé les parures qu'elle portait ; des troupes affligées assiègent les sanctuaires. Celles-ci arrosent les dieux de larmes; celles-là clouent leur poitrine sur le sol dur ; abattues, elles répandent sur le seuil sacré leurs chevelures arrachées et frappent de hurlements répétés des oreilles habituées à être invoquées avec des vœux. Et toutes ne se prosternèrent pas au temple du souverain Maître du tonnerre ; elles se partagèrent les dieux; aucun autel ne manqua de mères, soucieuses de ne pas susciter de jalousie. L'une d'elles s'écria, les joues humides et déchirées, les bras livides noircis par les coups : "Maintenant meurtrissez vos poitrines, malheureuses mères ; maintenant arrachez vos cheveux et ne différez pas ces marques de douleurs ; ne les conservez pas pour les malheurs suprêmes ; nous avons encore le droit de pleurer tant que la fortune des chefs est en suspens”. »

Le spectacle d'affliction que les matrones mettent en scène publiquement sur les escaliers conduisant aux temples ne diffère pas de celui des pleureuses décrites par Sénèque : elles pleurent, s'arrachent les cheveux, hurlent, se déchirent les joues et se frappent les bras et la poitrine. La comparaison se révèle d'autant plus efficace si l'on considère que même les supplicationes, selon B. Linke ${ }^{26}$, ont un effet cathartique : elles permettent d'articuler et de canaliser la peur collective. À la différence de l'émotion du deuil qui, au

23. Pour cette comparaison, voir aussi Sterbenc Erker, 2004, p. 283.

24. Linke, 2003, p. 71. Sur les supplicationes, voir aussi Naiden 2006.

25. Lucan., bell. civ., 2, 28-41 (trad. A. Bourgery, 1997, $2^{\mathrm{e}}$ édition revue et corrigée par Paul Jal, Les Belles Lettres, CUF, Paris, 1997).

26. Linke, 2003, p. 74. 
départ, ne concerne qu'une seule famille, la peur d'une catastrophe concerne d'emblée toute la communauté. Cependant, dans un cas comme dans l'autre, l'essentiel demeure le fait d'agir ensemble, en théâtralisant une émotion qui aide à retrouver un équilibre.

\section{3/ LE RITE SUSCITE D'AUTRES ÉMOTIONS}

Selon l'explication proposée jusqu'ici, le rite représente un aboutissement des émotions : il les canalise pour les contrôler et les soigner en communauté. Cependant, en observant les funérailles, on se rend compte que la mise en scène du deuil, tout en ayant un effet cathartique pour ceux qui l'accomplissent, a aussi pour fonction de susciter des sentiments nouveaux chez ceux qui l'observent.

Les funérailles romaines, on le sait, sont un spectacle ${ }^{27}$. La description que Polybe en donne est parlante. À propos de la laudatio funebris, que le fils du défunt prononce sur les rostres, il affirme ${ }^{28}$ :

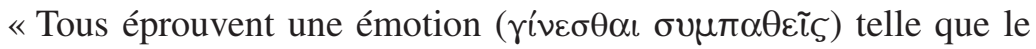
deuil cesse de paraître limité à la famille et devient celui du peuple tout entier ». Et ensuite, à propos des images des ancêtres qui défilent dans le convoi funèbre porté par des hommes, il constate ${ }^{29}:$ " Qui ne serait inspiré en voyant les images des hommes dont la valeur est glorieuse, toutes réunies, pour ainsi dire vivantes et animées ? Quel plus beau spectacle pourrait-on montrer? » De ce spectacle, les lamentations et le planctus font aussi partie. Par un passage de Varron $^{30}$ cité auparavant, on sait que les preaficae agissent devant la maison du mort : leur lamentation était donc visible pour l'ensemble de la communauté. En outre, un bas-relief d'Amiternum (fig. 3) représente un groupe de femmes qui accomplit des gestes de lamentation lors d'une pompa funebris : deux femmes précèdent le cadavre en portant leurs mains à la tête et aux cheveux alors que

27. Flaig, 2003, p. 51-59.

28. Polyb., 6, 53, 3 (texte établi par Raymond Weil avec la collaboration de Claude Nicolet, Les Belles Lettres, CUF, Paris, 1977).

29. $6,53,10$.

30. Varro, ling. $7,70$. 


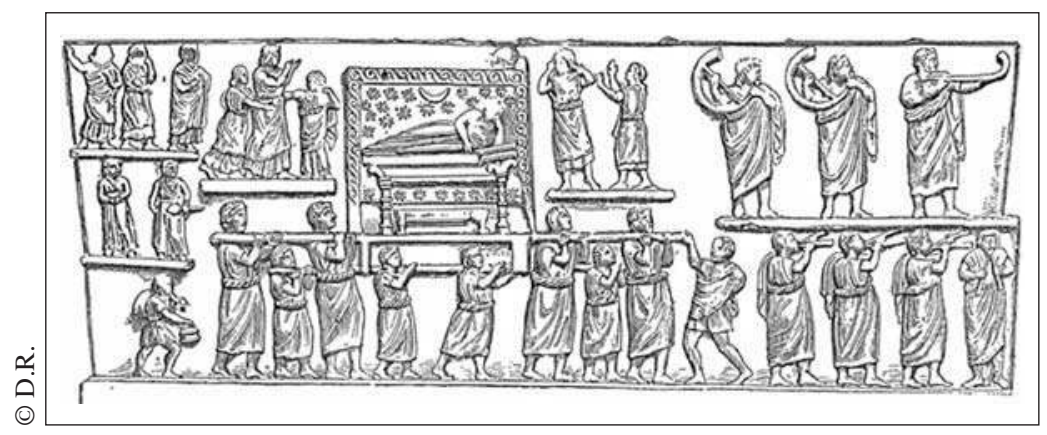

Fig. 3. Relief de Amiternum ( $2^{\mathrm{e}}$ moitié du $1^{\mathrm{er}}$ s. av. J.-C.), Museo Nazionale d'Abruzzo, L'Aquila.

(Dessin tiré de Ch. Daremberg, Edm. Saglio, Dictionnaire des Antiquités grecques et romaines, tome 2, $1896 \mathrm{sv}$ funus, p. 1392, fig. 3361).

d'autres le suivent en se frappant la poitrine. Elles aussi agissent devant un public qui les regarde.

Un public assiste donc aux rites du planctus et de la lamentation et écoute le discours accompli par les descendants de la famille. Ce spectacle peut susciter des émotions comme la compassion, mais aussi l'admiration pour une famille si riche qui peut se permettre tant d'effusions, dont certaines «payées », comme celles des praeficae. Des limitations, qui remontent aux Douze Tables (vers 450 av. J.-C.), visent à empêcher que le planctus soit trop violent et qu'on dépense trop d'argent pour des funérailles ${ }^{31}$. Cela révèle que le planctus, tout autant que les dépenses, était un moyen pour les familles importantes de l'aristocratie de s'illustrer aux yeux de la population qui se transformait, pour l'occasion, en public.

31. Cf. Cic., leg., 2, 59 : «Donc la dépense étant réduite à trois riciniae - coiffes de deuil -, un linceul pourpre et dix joueurs de flûtes, la loi supprime encore la lamentation excessive. "Que les femmes ne se râclent pas les joues et ne tiennent pas de lessus [« lamentation »] à l'occasion du convoi". [...] Voilà des mesures louables et communes aux riches comme à la plèbe, et cela est bien conforme à la nature de faire disparaître toute inégalité dans la mort » (texte établi et trad. par Georges de Plinval, Les Belles Lettres, CUF, Paris, 1968) ; cf. aussi Cic., leg., 2, 64 ; Serv., ad Aen., 12, 606 ; d'autres dispositions sont citées par Cic., leg., 2, 60 ; et aussi Engels, 1998, p. 166. 
Comme j'ai cherché à le montrer dans cette étude, le rapport entre les aspects affectif et rituel du deuil est complexe. Le rite est un instrument qui permet de transformer la dimension affective, personnelle et intime en un fait social et partagé par la communauté. Mais, il est aussi un phénomène qui impressionne le public et peut susciter des émotions de complainte et d'admiration. Ainsi les manifestations collectives et rituelles du deuil sont-elles simultanément un point de départ et d'arrivée des émotions.

Université de Lausanne, Anthropole, IASA, 1015 Lausanne

Francesca.PrescendiMorresi@unil.ch

Université de Genève, rue de Candolle 3 CH-1211 Genève 4

francesca.prescendi@lettres.unige.ch

\section{BIBLIOGRAPHIE CITÉE}

Borgeaud, Philippe, « Rites et émotions. Considérations sur les mystères », in J. Scheid (éd.), Rites et croyances dans les religions du monde romain, Entretiens sur l'Antiquité classique 53, Fondation Hardt, VandoeuvresGenève, 2007, 189-222.

Brigger, Eliane, Giovannini, Adalberto, «Prothésis : étude sur les rites funéraires chez les Grecs et chez les Étrusques », Mélanges de l'École Française de Rome, 116 (2004), 179-248.

CorbeILl, Anthony, Nature embodied. Gesture in Ancient Rome, Princeton, 2004.

DE Martino, Ernesto, Morte e pianto rituale. Dal lamento funebre antico al pianto di Maria, Torino, 1983 (= 1975).

ENGELs, Johannes, Funerum sepulcrorumque magnificentia. Begräbnis- und Grabluxusgesetze in der griechisch-römischen Welt mit einigen Ausblicken auf Einschränkungen des funeralen und sepulkralen Luxus im Mittelalter und in der Neuzeit, Stuttgart, 1998.

FlaIG, Egon, Ritualisierte Politik : Zeichen, Gesten und Herrschaft im Alten Rom, Göttingen, 2003.

Graver, Margaret, Cicero on the Emotions. Tusculan Disputations 3 and 4, Chicago, 2002.

GrAVER, Margaret, Stoicism and Emotions, Chicago, 2008 (à paraître).

KIEDORF, Wilhelm, Laudatio funebris. Interpretationen und Untersuchungen zur Entwicklung der römischen Leichenrede, Meisenheim am Glan, 1980.

Konstan, David, The emotions of the Ancient Greeks. Studies in Aristotle and Classical Literature, Toronto, 2006. 
KudLIEN, Fridolf, « Berufsmäßige Klageweiber in der Kaiserzeit », Rheinische Museum NF138 (1995), 177-187.

LINKE, Bernhard, « Emotionalität und Status : zur gesellschaftlichen Funktion von supplicationes und lectisternia in der römischen Republik », in A. Kneppe, D. Metzler, Die emotionale Dimension antiker Religiosität, Münster, 2003, 65-86.

NAIDEn, Fred S., Ancient Supplication, Oxford, 2006.

PERFIGLI, Michol, Indigitamenta. Divinità funzionali e funzionalità divina nella religione romana, Pisa, 2004.

PRESCENDI, Francesca, « Il lutto dei padri nella cultura romana », in F. Hinard (éd.), La mort au quotidien dans le monde romain, Actes du colloque organisé par l'Université de Paris IV (Paris, Sorbonne, 7-9 octobre 1993), De Boccard, Paris, 1996, 147-154.

PRESCENDI, Francesca, « Klagende Frauen. Zur weiblichen Trauerhaltung in Rom », in Th. Späth et B. Wagner-Hasel (éd.), Frauenwelten in der Antike. Geschlechterordnung und weibliche Lebenspraxis, Stuttgart, 2000, 102-111.

Riposati, Benedetto, M. Terenti Varronis, De vita populi Romani, Milano, 1939.

ScHEID, John, «Libitina, Lubentina, Venus Libitina et les morts », in S. Orlandi (éd.), Libitina e dintorni. Atti dell'VI Rencontre francoitalienne sur l'épigraphie : Libitina e i luci sepolcrali. Le leges libitinariae campane. Iura sepulcrorum : vecchie e nuove iscrizioni, Roma 2004, 13-19.

STERBENC ERKER, Darja, « Voix dangereuses et force des larmes : le deuil féminin dans la Rome antique », Revue de l'histoire des religions, 221 (2004), 259-291.

Wesch-Klein, Gabriele, Funus publicum. Eine Studie zur öffentlichen Beisetzung und Gewährung von Ehrengräbern in Rom und den Westprovinzen, Stuttgart, 1993. 